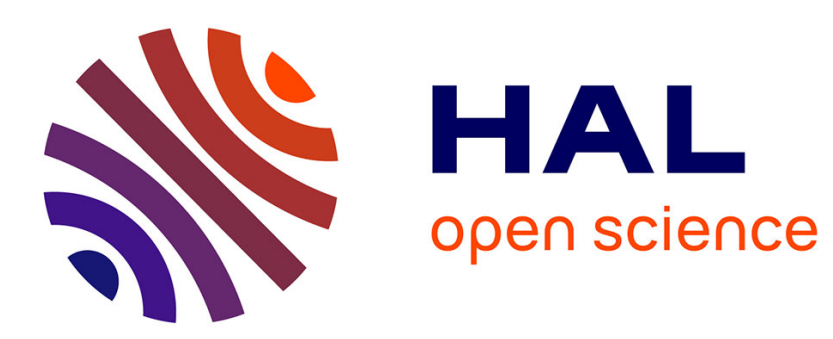

\title{
Towards Seamless Interaction between Physical and Virtual Locations for Asymmetric Collaboration
}

\author{
Damien Clergeaud, Joan Sol Roo, Martin Hachet, Pascal Guitton
}

\section{To cite this version:}

Damien Clergeaud, Joan Sol Roo, Martin Hachet, Pascal Guitton. Towards Seamless Interaction between Physical and Virtual Locations for Asymmetric Collaboration. VRST 2017 - 23rd ACM Symposium on Virtual Reality Software and Technology, Nov 2017, Gothemburg, Sweden. pp.1-5, 10.1145/3139131.3139165. hal-01649262

\section{HAL Id: hal-01649262 https://hal.science/hal-01649262}

Submitted on 27 Nov 2017

HAL is a multi-disciplinary open access archive for the deposit and dissemination of scientific research documents, whether they are published or not. The documents may come from teaching and research institutions in France or abroad, or from public or private research centers.
L'archive ouverte pluridisciplinaire HAL, est destinée au dépôt et à la diffusion de documents scientifiques de niveau recherche, publiés ou non, émanant des établissements d'enseignement et de recherche français ou étrangers, des laboratoires publics ou privés. 


\section{Towards Seamless Interaction between Physical and Virtual Locations for Asymmetric Collaboration}

\author{
Damien Clergeaud \\ Inria \\ Bordeaux, France \\ damien.clergeaud@inria.fr \\ Martin Hachet \\ Inria \\ Bordeaux, France \\ martin.hachet@inria.fr
}

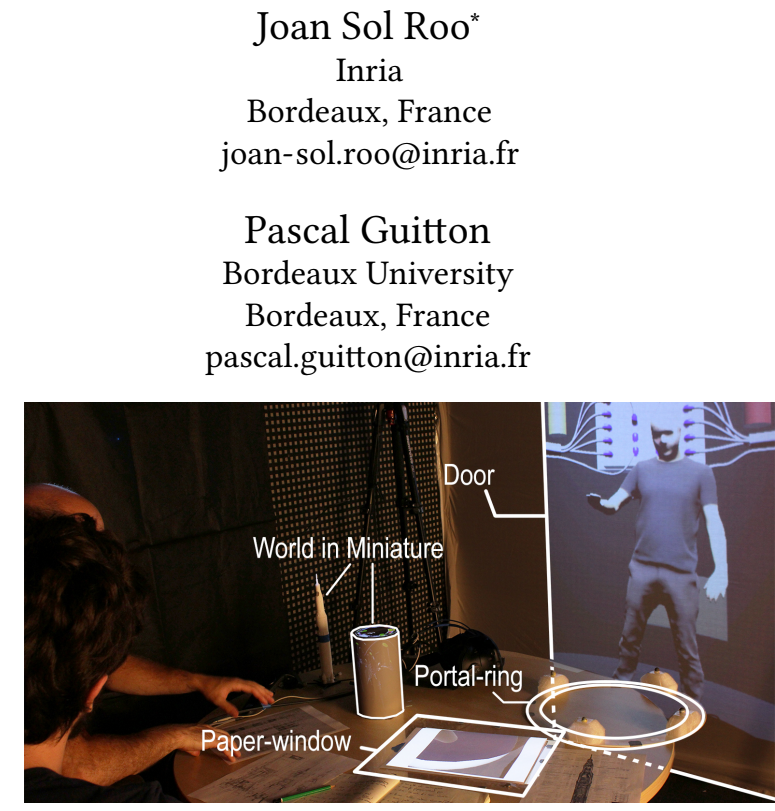

Figure 1: The collaboration between a physical meeting room and a digital mock-up is supported thanks to different artefacts that provide users different degrees of immersion into the remote scene.

\section{INTRODUCTION}

In the industrial context, Virtual Reality (VR) is more and more used to support the design and iteration of products (CAD reviews, ergonomic study, assembly task design, among many others). Compared to physical prototypes, virtual environments enable engineers to test solutions faster, safer, and cheaper. Moreover, in some domains such as in the case of the aerospace industry, all the products are designed digitally with CAD software, which facilitates the use of VR. On the other hand, the design of complex artefacts involve multiple iterations based on decisions discussed by multiple stakeholders, which still require physical meetings.

The need of both physical meetings and VR prototyping leads to asymmetric collaboration. Indeed, the combination of both physical and virtual spaces could not be replaced by co-locating all the participants: immersing all the users in a virtual environment would break the meeting dynamics, while keeping the operators in the meeting room would greatly reduce the experimentation possibilities. As a result, collaborators with different roles at different locations must effectively communicate in order to successfully achieve their objectives and sometimes have to move between virtual and physical spaces.

The question regarding asymmetric collaboration was brought to us by engineers from Airbus Group, a mayor aerospace manufactoring company. They use VR to design assembly tasks directly with 
the final operator immersed in the digital mockup, while experts supervise and guide the process from a meeting room. In order to follow the operator's work, experts can observe him or her through a monitor located at the meeting room, while an audio channel supports the communication between the two locations. For instance, experts could design a procedure to mount a particular system in the launcher while the operator is following the procedure to complete its design.

The engineers from three different branches of Airbus Group agreed on limitations of the current approach for asymmetric collaboration. Their main concerns orbit around the fact that the viewpoint and the audio channel do not support awareness and communication properly. First, it is not uncommon that the experts forget the presence of the operator, since they work in parallel. Second, they struggle to provide instructions for precise or complex tasks. Finally, there are instances where being temporarily present at the remote location (in either direction) could ease the understanding between participants.

The purpose of this work is to provide tools that address the limitations of the current approaches for asymmetric collaboration, in order to ease the communication between these remote locations. This involves i) supporting awareness between spaces, ii) tools that support remote operations, and iii) ways to visit the remote location. To do so, we choose to focus on the creation of Through-The-Lens technique (TTL) artefacts for both the physical and virtual locations.

The contributions of the current work are (Figure 1): 1) the conception and prototyping of tangible Mixed Reality lenses - and their virtual counterparts - for asymmetric collaboration, 2) the description of the system that supports these artefacts, and 3) preliminary feedback from future end users of the aerospace industry.

\section{RELATED WORK}

This work is at the intersection between 1) TTL for Virtual Reality, 2) linking physical and digital information, and 3) tele-presence technologies. This section briefly explore these research areas.

Through-The-Lens techniques (TTL) allow connecting spaces together. More formally, TTL enable users to simultaneously explore a virtual environment from two or more different viewpoints as described by Stoev et al. [Stoev and Schmalstieg 2002]. If the second viewpoint is linked to the location to another immersed user, it provides a new channel for communication, as proposed by Kunert et al. with Photoportals [Kunert et al. 2014]. Photoportals are a mix between viewports and portals with a collaborative purpose, enabling users to create flat (2D) or volumetric (3D) viewports to observe a remote location or to directly teleport themselves through it. It can also be used to retrieve remote objects, reducing the perceived distance between locations.

Instead of bringing the user inside the virtual space, it is possible to merge virtual and physical environments, notably through the use of projection [Bimber and Raskar 2006]. The Office of the Future [Raskar et al. 1998] envisioned to turn everyday spaces into CAVEs, which was then implemented using Spatial Augmented Reality (SAR) [Jones et al. 2014]. This technique enables the creation of interactive augmented objects and tools. Even when SAR has limited display capabilities, they can be complemented using seethrough devices [Benko et al. 2015; Billinghurst et al. 2001]. Another complementary way of bridging the distance between physical and digital spaces are Tangible User Interfaces (TUIs) [Fishkin 2004], which provide physical handles to digital information. TUIs can be used in combination with screens (as with the classical example from Hinckley et al. [Hinckley et al. 1994]), in VR spaces (as with Specimen Box [Zielinski et al. 2017]), or in combination with SAR (physical-virtual tools from Marner et al. [Marner et al. 2009], among many others). As a result, users can experience embodied interaction with digital content.

Thanks to the advances in sensing technologies, remote physical elements and locations can be digitalized [Orts-Escolano et al. 2016], extending the reach of the previous tools from purely digital content to any digitalized content. In this line of work, researchers are exploring ways to combine physical and digital. Examples include the digital navigation of the physical environment [Komiyama et al. 2017], asymmetric views of a digital scene [Ibayashi et al. 2015] or combination of both [Roo and Hachet 2017a,b].

Close in spirit to this work is the interactive tangible window [Angelini et al. 2016], which combines TTL, physical supports and telepresence. It was designed to ease the use of video-call applications for eldery adults. Users can then interact with remote locations, making the distance disappear thanks to the interface, while the interaction is framed into the physical environment.

To summarise, much effort have been done to combine physical and digital, locally and remotely. We propose to extend this line of research by supporting the asymmetrical collaboration between physical and digital spaces, using both traditional VR and tangible metaphors, as described in the next section.

\section{PROPOSED METHOD}

In order to facilitate the asymmetric collaboration between physical and virtual locations, we propose to support three core features (Figure 2): 1) awareness through overview of the remote scene and collaborators; 2) the ability to remotely interact and manipulate elements; and 3) the capability to navigate between locations.

With these objectives in mind, we created artefacts that support the required features, using either SAR or VR. SAR and TUIs were used in the instances where immersion would break the dynamics of the local interaction. For the virtual locations, the interaction supports both tangible and purely virtual artefacts; these alternatives were created in order to enable more immersion or to free the

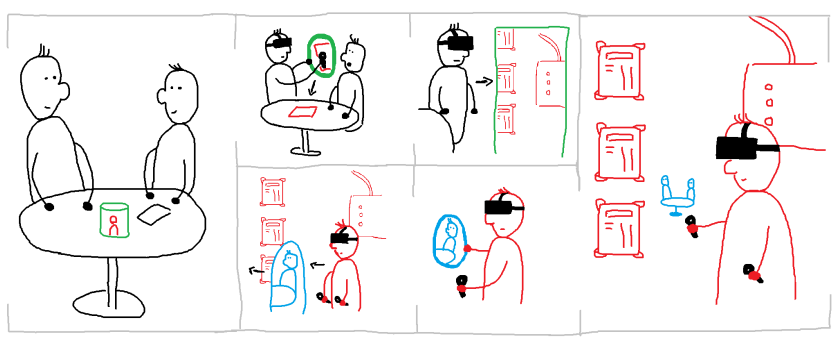

Figure 2: The artefacts bridge the distance between the local and remote locations, enabling progressive immersion and interaction on the other side, up to the point of going there. 
hands respectively. All the interaction was supported by extending a previous project from the same authors [Roo and Hachet 2017a].

\subsection{Awareness through Scaled Representations}

In order to enable the communication between the two locations, it is first necessary to have an overall understanding of the whole space and the location of each collaborator. For this reason, we decided to use World-In-Miniature [Stoakley et al. 1995] as the starting point for interaction and communication. WIMs provide an overview of the context of the remote collaborators, while also supporting coarse interaction through pointing. Collaborators are displayed using avatars (rigged 3D scans [Feng et al. 2015]).

We implemented independent scaled down mock-ups for physical and virtual locations. The physical counterpart is supported by a physical mock-up (which is possible to $3 \mathrm{D}$ print thanks to the already available CAD information) with spatial augmentation generated via projection. Spatially augmented mock-ups work as ambient displays [Wisneski et al. 1998], its always-available subtle information makes them ideal to keep the users aware of the remote activity without being too distracting. In VR, the WIM displays the remote table and its surroundings, immersed users can hold a tangible prop to observe the remote place, and leave the prop on the workbench nearby. Alternatively, immersed users can interact with a virtual WIM (i.e., without a physical support).

When building physical mock-ups, both limitations and opportunities arise given the intrinsic physical properties. In the case of the launcher mock-up, two complementary views are necessary: an overview of the whole launcher and a detailed overview of the working piece. This was reproduced in our case with two separated mock-ups (Figure 3-left): the first one showing the location of the active section, while the second one shows combined inside-out and top-down views of the active room.

\subsection{Interaction through Windows}

Windows provide lenses to other spaces from given points of view, enabling interaction through them, as with traditional desktop screens. The main advantage of windows and their relative small size is the possibility of using several of them simultaneously.

We support two types of windows: interactive paper and portalrings. The former provides a see-through view of the scene, allowing to display mid air information at the meeting room location much like a see-through tablet, as provided by [ANONYMOUSPaper]. Portal-rings are hollow frames that display the remote location, and enable interaction through them. Users can pass their hands to interact with the other side, and move elements between locations (Figure 3-center). Windows then provide the possibility to observe and interact with the other side, allowing a better communication between locations.

\subsection{Navigation through Doors}

Doors are similar to windows, but besides providing a viewpoint of the remote location, they also enable to go there. Doors and teleportation are fairly common in VR, in particular to deal with limited physical spaces. In our context, we place a virtual door at a physical wall of the meeting room thanks to projection (Figure 3right). Regarding the navigation, the user immersed in VR does not need additional tools, as portals are a commonly used technique. For the users at the meeting room, the door is displayed on a wall using projection while the navigation is performed in VR, by wearing an HMD. Alternatively, the door could be located on a nearby space with CAVE capabilities, thus the process of walking to the door could provide a seamless transition into a dynamic VR space.

Once users transited to the remote location, they can directly interact with its virtual version. Displaying tele-presence collaborators in VR is done by showing their avatar, while in the meeting room is necessary to use an additional display.

\section{SYSTEM IMPLEMENTATION}

The prototype was implemented as an extension of [Roo and Hachet 2017a]. It is comprised of peer applications connected using UDP and OSC (real-time control protocol built over UDP). In each location, the hardware comprised of Optitrack Flex cameras, HTC vive, and optionally projectors. Both applications are identical, except by the hardware configuration; this design decision supports the creation of a topology of locations interconnected through artefacts, yet the artefact configuration and networking require further consideration. The audio communication was not explored for the current version of the system.

\section{FEEDBACK FROM PARTNERS}

After the design and implementation of the prototype, we performed a demo session with the three engineers that brought the use case to us. The engineers were presented with the features of the system one by one, following the structure of this paper, from the meeting room side (since the VR interaction is closer to their expertise); one of the researchers was immersed inside the launcher mock-up from a nearby room.

The physical WIM was perceived as a good way to be aware of the remote location, placing the operator literally at the center of the table. The use of 3D printing created mixed emotions, and the conclusion is that their utilisation will depend of the use case (e.g., good for training since it is reused, not good for daily meetings). The manipulation of the WIM while immersed was enjoyed yet rapidly discarded as impractical given its weight, preferring the use of the pure virtual WIM.

Regarding the portal-ring, the engineers found the possibility of taking objects from the other location of great use, and the gesture of bringing the object through the portal was intuitive. On the other hand, holding the ring was once again seen as unpractical, and they preferred the virtual version. The use of paper-windows was rapidly understood and they envisioned this the easiest to adopt through the use of tracked tablets. This was particularly appreciated when interacting with the WIM in particular, and more generally with all the digital objects, like the ones taken through the portal-ring.

The door was the most appreciated artefact, since it allows the operator to come into the meeting room, while the navigation into the other direction was not consider novel. When presented with the door, they envisioned how to improve their current workflow using it. Concretely, operators follow strict step-by-step protocols to prevent mistakes, so they need to keep the virtual scene uncluttered; being able to do back and forths to the meeting room enables 

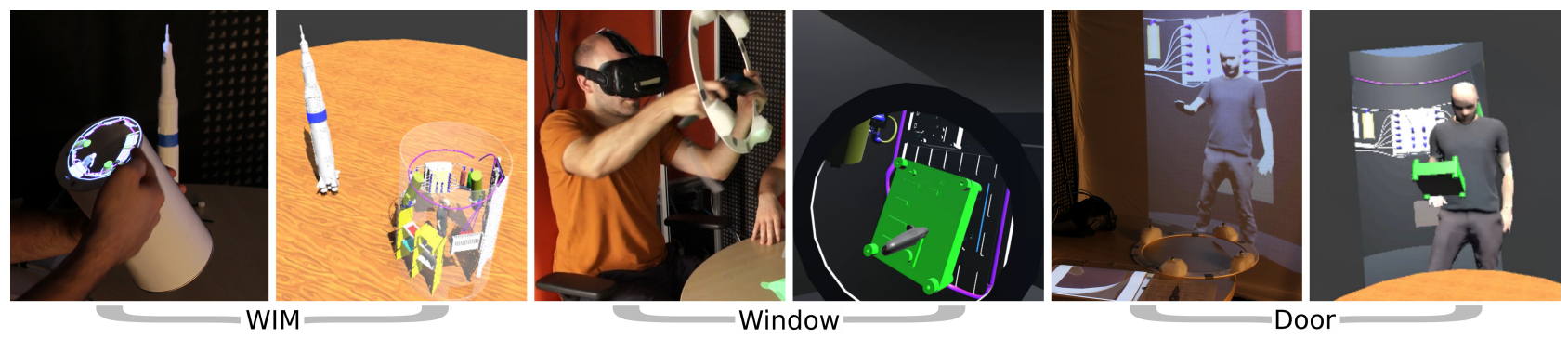

Figure 3: Pictures and virtual views of the artefacts from the meeting room.

them to focus on one task at a time, while the experts can coordinate the whole process. In their context, the use of the door to move elements between locations was better suited than portal-rings.

Regarding the technologies involved, the engineers mentioned limitations on both. The VR helmet was perceived as cumbersome to utilise and equip. They considered the extension of the system to support a lighter augmented reality helmet such as Hololens, while they also were aware of the loss of resolution and FOV implied. Regarding SAR, on one hand they liked the un-instrumented interaction, yet the use of flat augmented surfaces was limited, and they would prefer richer rendering. An interesting conclusion they reached is that not all experts will use the same modalities, and the limitations of one technology can be mitigated with the other.

\section{CONCLUSION}

This work was inspired by the current limitations in asymmetric collaboration expressed by industrial engineers. Based on the literature, we created artefact prototypes to improve the awareness, communication and interaction between locations. Finally, we presented these prototypes to engineers in order to get feedback.

From the proposed solutions, the use of windows and doors where the two preferred features. Windows were considered to complement the current approach, while also being easy to adopt. Regarding the doors, they envisioned how they could improve the existing workflows. Interestingly, the tangible physical TTLs were instantly understood, yet quickly discarded for practical reasons. We consider this does not suggest that tangibles should be avoided when designing prototypes, quite the opposite. Their use in early stages can lead to a better understanding of the interaction metaphors, to then be replaced with purely virtual widgets.

We consider that the proposed interaction techniques and artefacts can be extended to other contexts, and are not limited to SAR-VR collaboration. Indeed, the presented interactions could be used between any two locations, each of them either physical (digitalized) or virtual. Our conclusion is that, instead of trying to provide a single space that supports all the interactions, it is preferable to have several spaces with specific aims, even when these locations are only virtually separated.

\section{ACKNOWLEDGMENTS}

This work was made possible thanks to Airbus Group, and the ISAR project (ANR-14-CE24-0013).

\section{REFERENCES}

Leonardo Angelini, Francesco Carrino, Maurizio Caon, Frédéric Lemaréchal, Nadine Couture, Omar Abou Khaled, and Elena Mugellini. 2016. Testing the tangible interactive window with older adults. GeroPsych (2016).

Hrvoje Benko, Eyal Ofek, Feng Zheng, and Andrew D Wilson. 2015. Fovear: Combining an optically see-through near-eye display with projector-based spatial augmented reality. In UIST '15. ACM, 129-135.

Mark Billinghurst, Hirokazu Kato, and Ivan Poupyrev. 2001. The magicbook-moving seamlessly between reality and virtuality. IEEE CGandA 21, 3 (2001).

Oliver Bimber and Ramesh Raskar. 2006. Modern approaches to augmented reality. In ACM SIGGRAPH 2006 Courses. ACM, 1.

Andrew Feng, Dan Casas, and Ari Shapiro. 2015. Avatar Reshaping and Automatic Rigging Using a Deformable Model. In Proceedings of the 8th ACM SIGGRAPH Conference on Motion in Games (MIG '15). ACM, New York, NY, USA, 57-64.

Kenneth P Fishkin. 2004. A taxonomy for and analysis of tangible interfaces. Personal and Ubiquitous Computing 8, 5 (2004), 347-358.

Ken Hinckley, Randy Pausch, John C Goble, and Neal F Kassell. 1994. A threedimensional user interface for neurosurgical visualization. In Proc. of the SPIE Conference on Medical Imaging. 126-136.

Hikaru Ibayashi, Yuta Sugiura, Daisuke Sakamoto, Natsuki Miyata, Mitsunori Tada, Takashi Okuma, Takeshi Kurata, Masaaki Mochimaru, and Takeo Igarashi. 2015. Dollhouse VR: a multi-view, multi-user collaborative design workspace with VR technology. In SIGGRAPH Asia 2015 Emerging Technologies. ACM, 8.

Brett Jones, Rajinder Sodhi, Michael Murdock, Ravish Mehra, Hrvoje Benko, Andrew Wilson, Eyal Ofek, Blair MacIntyre, Nikunj Raghuvanshi, and Lior Shapira. 2014. RoomAlive: magical experiences enabled by scalable, adaptive projector-camera units. In UIST. ACM, 637-644.

Ryohei Komiyama, Takashi Miyaki, and Jun Rekimoto. 2017. JackIn Space: Designing a Seamless Transition Between First and Third Person View for Effective Telepresence Collaborations. In Proceedings of the 8th Augmented Human International Conference (AH '17). ACM, New York, NY, USA, Article 14, 9 pages.

André Kunert, Alexander Kulik, Stephan Beck, and Bernd Froehlich. 2014. Photoportals: shared references in space and time. In CSCW'14. ACM.

Michael R Marner, Bruce H Thomas, and Christian Sandor. 2009. Physical-virtual tools for spatial augmented reality user interfaces. In ISMAR, Vol. 9. 205-206.

Sergio Orts-Escolano, Christoph Rhemann, Sean Fanello, Wayne Chang, Adarsh Kowdle, Yury Degtyarev, David Kim, Philip L Davidson, Sameh Khamis, Mingsong Dou, and others. 2016. Holoportation: Virtual 3D Teleportation in Real-time. In UIST '16. ACM, 741-754.

Ramesh Raskar, Greg Welch, Matt Cutts, Adam Lake, Lev Stesin, and Henry Fuchs. 1998. The office of the future: A unified approach to image-based modeling and spatially immersive displays. In SIGGRAPH'98. ACM, 179-188.

Joan Sol Roo and Martin Hachet. 2017a. One Reality: Augmenting How the Physical World is Experienced by combining Multiple Mixed Reality Modalities. In UIST'17. ACM, Quebec City, Canada.

Joan Sol Roo and Martin Hachet. 2017b. Towards a hybrid space combining Spatial Augmented Reality and virtual reality. In 3DUI'17. IEEE, 195-198.

Richard Stoakley, Matthew J. Conway, and Randy Pausch. 1995. Virtual Reality on a WIM: Interactive Worlds in Miniature. In CHI '95 (CHI '95). ACM Press/AddisonWesley Publishing Co., New York, NY, USA, 265-272.

Stanislav L Stoev and Dieter Schmalstieg. 2002. Application and taxonomy of throughthe-lens techniques. In VRST. ACM, 57-64.

Craig Wisneski, Hiroshi Ishii, Andrew Dahley, Matt Gorbet, Scott Brave, Brygg Ullmer, and Paul Yarin. 1998. Ambient displays: Turning architectural space into an interface between people and digital information. In International Workshop on Cooperative Buildings. Springer, 22-32.

David J Zielinski, Derek Nankivil, and Regis Kopper. 2017. Specimen Box: A tangible interaction technique for world-fixed virtual reality displays. In 3DUI'17. IEEE, $50-58$. 